\title{
Inhibition of vascular endothelial growth factor A expression in mouse granulosa cells by lentivector-mediated RNAi
}

\author{
N. Ally, X.L. Zou, B.C. Jiang, L. Qin, L. Zhai, P. Xiao and H.L. Liu \\ Department of Animal Breeding and Genetics, \\ College of Animal Science and Technology, \\ Nanjing Agricultural University, Nanjing, China \\ Corresponding author: H.L. Liu \\ E-mail: liuhonglin@263.net
}

Genet. Mol. Res. 11 (4): 4019-4033 (2012)

Received May 4, 2012

Accepted July 19, 2012

Published November 28, 2012

DOI http://dx.doi.org/10.4238/2012.November.28.1

\begin{abstract}
Vascular endothelial growth factor (VEGF) has been found responsible for the induction of proliferation and differentiation in granulosa cells. We constructed four short hairpin RNA (shRNA) expression plasmids targeting the mouse VEGFA gene, and examined their effect on VEGF expression in mouse granulosa cells (MGC) in vitro. Four different shRNA oligonucleotides targeting the coding sequence of mouse VEGFA mRNA and one negative control (shNC) were designed and cloned into a pGPU6/GFP/Neo siRNA expression vector, and transiently transfected into MGC. At $48 \mathrm{~h}$ post-transfection, total RNA was extracted from the cells and subjected to qRT-PCR analysis. The most effective interference vector, shVEGF1487 was chosen for lentiviral construction. The recombinant plasmid was then transfected into 293FT cells via Lipofectamine ${ }^{\mathrm{TM}}$ 2000-mediated gene transfer, for the production of lentivirus, and then concentrated via ultracentrifugation. This lentiviral vector was then used for the transduction of MGC. VEGFA gene expression, apoptosis genes and VEGFA receptor genes were detected by qRT-PCR, the VEGFA
\end{abstract}


protein level in culture media by ELISA assay and protein levels in MGC by Western blot analysis. The four VEGFA expression plasmids were successfully constructed and the most effective interference vector, shVEGF1487, was chosen for lentiviral production and MGC transduction. There was significant knockdown of the VEGFA gene, receptor genes and apoptosis genes for all the shVEGF constructs, compared with the shNC and Mock controls. The lentiviral vector also gave significant knockdown of the VEGFA gene. Protein levels were lower for most of the shVEGFs based on Western blot analysis with exception of VEGF1359; in this case, it was higher than shNC but lower than for the Mock group. Lentivector-transduced MGC also gave lower levels of protein. We conclude that shVEGF expression plasmids and lentivector carrying RNAi are promising tools for the inhibition of VEGF, the corresponding receptor genes, and apoptosis gene expression in MGC.

Key words: VEGF; siRNA; shRNA; MGC; Apoptosis; Gene and protein expression

\section{INTRODUCTION}

Over the last two decades, the vascular endothelial growth factor (VEGF) family and its receptors have been well characterized and are part of the most important signaling pathway in angiogenesis. Seven family members have been identified, VEGFA, VEGFB, VEGFC, VEGFD, VEGFE, and placental growth factor (PIGF1 and PIGF2), all of which signal through three tyrosine kinase receptors, VEGFR-1 (Flt-1, fms-induced tyrosine kinase receptor), VEGFR-2 (Flk-1, fetal liver kinase; KDR, kinase insert domaincontaining receptor) and VEGFR-3 (Flt-4) (Klagsbrun and D'Amore, 1996; Terman and Dougher-Vermazen, 1996; Ferrara, 2002; Hicklin and Ellis, 2005). VEGFA is the most prominent and biologically active member of the VEGF family and is thought to play a pivotal role in the regulation of angiogenesis in the ovary (Tamanini and De Ambrogi, 2004) and granulosa cells. Its expression in ovarian follicles depends on follicular size. Previous research with bovine and porcine follicles has shown that VEGFA is weakly expressed during early ovarian follicular development and becomes more pronounced in granulosa and theca cells, along with dominant follicle development (Barboni et al., 2000; Greenaway et al., 2004). The rat ovary also shows similar characteristics, with some secondary follicles displaying extremely strong VEGFA immunoreactivity in the zona pellucida (Celik-Ozenci et al., 2003).

RNA interference (RNAi) is a sequence-specific post-transcriptional gene silencing mechanism, which is initiated by double-stranded RNA (dsRNA) of $\sim 21$ nucleotides long, and causes degradation of messenger RNA (mRNA) homologous in sequence to the dsRNA (Elbashir et al., 2001, 2002; Hannon, 2002; McManus and Sharp, 2002; Shi, 2003). Plasmidbased systems using RNA polymerase III, such as $\mathrm{H1}$ and U6, promoters to drive short hairpin RNA (shRNA) molecules, were established to stably produce short interfering RNA (siRNA) (Brummelkamp et al., 2002; Lee et al., 2002; Paul et al., 2002). These promoters are easily 
cloned and placed into vectors of limited carrying capacity, because of their short lengths of few hundred base pairs. These transcription initiation and termination sites are well defined, facilitating accurate shRNA design (Giering et al. 2008). These siRNAs are transcribed as short hairpin precursors ( $\sim 70$ nucleotides), generated by the cellular enzymes Dicer and Drosha, to yield active 21-nucleotide RNAs that recognize target mRNAs via base-pairing interactions. Afterwards, mature siRNAs and miRNAs (micro-RNAs) associate with proteins to create an RNA-induced silencing complex (RISC) (Wolters and MacKeigan, 2008). The mechanism for this selection is not yet fully understood, but it is thought that once a strand is selected and loaded into RISC, the other strand is destroyed (Petersen et al., 2006).

Recent studies have shown that inhibition of VEGF in vivo and in vitro causes blockade of neovascularization and decreases granulosa and theca cell proliferation in the rat (Abramovich et al., 2010) and primate (Wulff et al., 2002), while improving follicular ultrastructural integrity and promoting follicular growth in goat ovary (Bruno et al., 2009). VEGF alone does not have an effect on cell proliferation and apoptosis, but this factor intensifies the cytoprotective effect of FSH and estradiol in granulosa cells in culture (Irusta et al., 2010). VEGF and its receptor VEGFR-2 play important roles in mediating and/or enhancing the effects of gonadotropins on granulosa cells (Doyle et al., 2010). Research shows that RNAi of VEGF using a plasmid-based strategy can stably silence VEGF expression in keloid fibroblasts (Zhang et al., 2008), decrease tumor vascularity and growth in vivo (Shen et al., 2007) and inhibit the growth of murine ovarian cancer cell lines in vitro (Zhang et al., 2003). Using oncolytic adenovirus to express shRNA also has antitumor effects through inhibition of VEGF expression (Yoo et al., 2007). In this study, we used, for the first time, a lentivector-mediated RNAi system, to construct four cellspecific shRNA expression plasmids targeting the mouse VEGFA gene to inhibit VEGFA in vitro in mouse granulosa cells (MGC). We also assessed their effects on the expression of the VEGFA gene, the receptor genes VEGFR-1 and VEGFR-2, and apoptosis genes at the mRNA and protein levels. We further used the most effective shRNA to construct the lentivirus and check its effect on VEGFA mRNA and protein levels.

\section{MATERIAL AND METHODS}

\section{Construction of shRNA plasmid}

The mRNA sequence of mouse VEGFA (Mus musculus, VEGFA, NM_009505) was sent to the Dharmacon siDESIGN Center (http://www.dharmacon.com/sidesign/) to design siRNAs. These siRNAs were selected on the basis of the ranking criteria described previously by Reynolds et al. (2004). Four siRNA target sequences were selected for this study (Table 1). One negative control siRNA containing a scramble sequence with the same nucleotide composition was also selected and termed shNC. A 9-nucleotide loop (TTCAAGAGA) was chosen for all the shRNAs. The Vienna RNA Secondary Package (http://www.tbi.univie.ac.at/ cgi-bin/RNAfold.cgi) was used to predict the secondary structure of the antisense strand of the shRNA. The shRNA-annealed oligonucleotides were ligated into pGPU6/GFP/Neo siRNA expression vectors between the BbsI and BamHI sites by T4 DNA ligase (TaKaRa), where these shRNA constructs were made by GenePharma. All constructs were verified by restriction and sequence analysis. 
Table 1. Hairpin short interfering RNA (siRNA) inserts.

\begin{tabular}{|c|c|c|}
\hline siRNA & Sequence (loop in bold letters) & Location \\
\hline VEGFA1359 & $\begin{array}{l}\text { CACCGCCAGCACATAGGAGAGATGATTCAAGAGATCATCTCTCCTATGTGCTGGCTTTTT } \\
\text { TGGATCCGGTCGTGTATCCTCTCTACTAAGTTCTCTAGTAGAGAGGATACACGACCGAAA } \\
\text { AAACCTAG }\end{array}$ & $1359-1379$ \\
\hline VEGFA1462 & $\begin{array}{l}\text { CACCGCGGAGAAAGCATTTGTTTGTTTCAAGAGAACAAACAAATGCTTTCTCCGCTTTTTTG } \\
\text { CGCCTCTTTCGTAAACAAACAAAGTTCTCTTGTTTGTTTACGAAAGAGGCGAAAAAACCTAG }\end{array}$ & $1462-1482$ \\
\hline VEGFA1051 & $\begin{array}{l}\text { CACCGACCCTGGCTTTACTGCTGTATTCAAGAGATACAGCAGTAAAGCCAGGGTCTTTTTTG } \\
\text { CTGGGACCGAAATGACGACATAAGTTCTCTATGTCGTCATTTCGGTCCCAGAAAAAACCTAG }\end{array}$ & $1051-1071$ \\
\hline VEGFA1487 & $\begin{array}{l}\text { CACCGATCCGCAGACGTGTAAATGTTTCAAGAGAACATTTACACGTCTGCGGATCTTTTTTG } \\
\text { CTAGGCGTCTGCACATTTACAAAGTTCTCTTGTAAATGTGCAGACGCCTAGAAAAAACCTAG }\end{array}$ & $1487-1507$ \\
\hline Scramble & $\begin{array}{l}\text { CACCGTTCTCCGAACGTGTCACGTCAAGAGATTACGTGACACGTTCGGAGAATTTTTTG } \\
\text { CAAGAGGCTTGCACAGTGCAGTTCTCTAATGCACTGTGCAAGCCTCTTAAAAAACCTAG }\end{array}$ & \\
\hline
\end{tabular}

The sequence of each insert is shown here as the form of double-strand, and underlined letters mean the sense sequence and anti-sense sequence of each siRNA.

\section{Laboratory animals and granulosa cell culture}

All animal procedures for these experiments were approved by the Nanjing Agricultural University Institutional Animal Care and Use Committee. Mice, 21 days old, were purchased from Qing Long Shan Co., China, and housed in the laboratory on a 14-h light/10-h dark cycle for 1 week and then treated with 10 IU pregnant mare serum gonadotropin (Ningbo Sansheng, China) to stimulate preovulatory follicular development. After $44-48 \mathrm{~h}$, the mice were killed and the ovaries removed and punctured with a $0.5-\mathrm{mm}$ diameter needle to release MGC, which were collected in DMEM/F12 (Gibco) with 10\% fetal bovine serum (Gibco) and medium supplements. Granulosa cells were cultured in $75-\mathrm{mL}$ flask with DMEM/F12 for 5-6 days in $5 \% \mathrm{CO}_{2}$ at $37^{\circ} \mathrm{C}$. The medium was then discarded and the cells washed twice with PBS to remove unattached cells. Trypsin was added at $2 \mathrm{~mL}$ per flask, and after $2 \mathrm{~min}$ at $37^{\circ} \mathrm{C}$, MGC were harvested. Cells were seeded $\left(1 \times 10^{5}\right.$ cells/well) on 6-well plates (Costar) and cultured in DMEM/F12 without antibiotics to achieve greater than $90 \%$ confluence on the day of transfection.

\section{Transfection of MGC with shRNA plasmids}

For transfection, $4 \mu \mathrm{g}$ each shRNA expression construct was used per well of a 6-well plate. Lipofectamine $2000^{\mathrm{TM}}$ (Invitrogen) was used as the transfection reagent at a ratio of $1 \mu \mathrm{g}$ DNA:1.5 $\mu \mathrm{L}$ Lipofectamine, according to manufacturer instructions. Transfected cells with transfecting reagent alone, termed Mock, and shNC-scrambled shRNA vector were used as controls. Lipofectamine/ DNA complexes were removed after $6 \mathrm{~h}$ and replaced with fresh medium with antibiotics.

\section{RNA isolation and quantitative real-time PCR analysis (qRT-PCR)}

Total RNA was isolated from cultured cells $48 \mathrm{~h}$ after transfection using the Trizol ${ }^{\circledR}$ reagent (Invitrogen). RNA was converted to cDNA using oligo(dT) primers and M-MLV Reverse Transcriptase (Promega) according to manufacturer instructions. Primers (Table 2) were designed for VEGFA, VEGF receptor genes Flt-1 and Flk-1, and apoptosis genes (Bax, FasL, TRAIL, and FoxO4), across introns to prevent amplification of genomic DNA, using the Primer 3 software. FastStart Universal SYBR Green Master (Roche) was used to perform qRT-PCR (ABI PRISM 7300) following the manufacturer protocol. The RNA 
expression of genes of interest was determined by normalization of the threshold cycle of these genes to that of the control GAPDH and displayed as the percentage change relative to the control group.

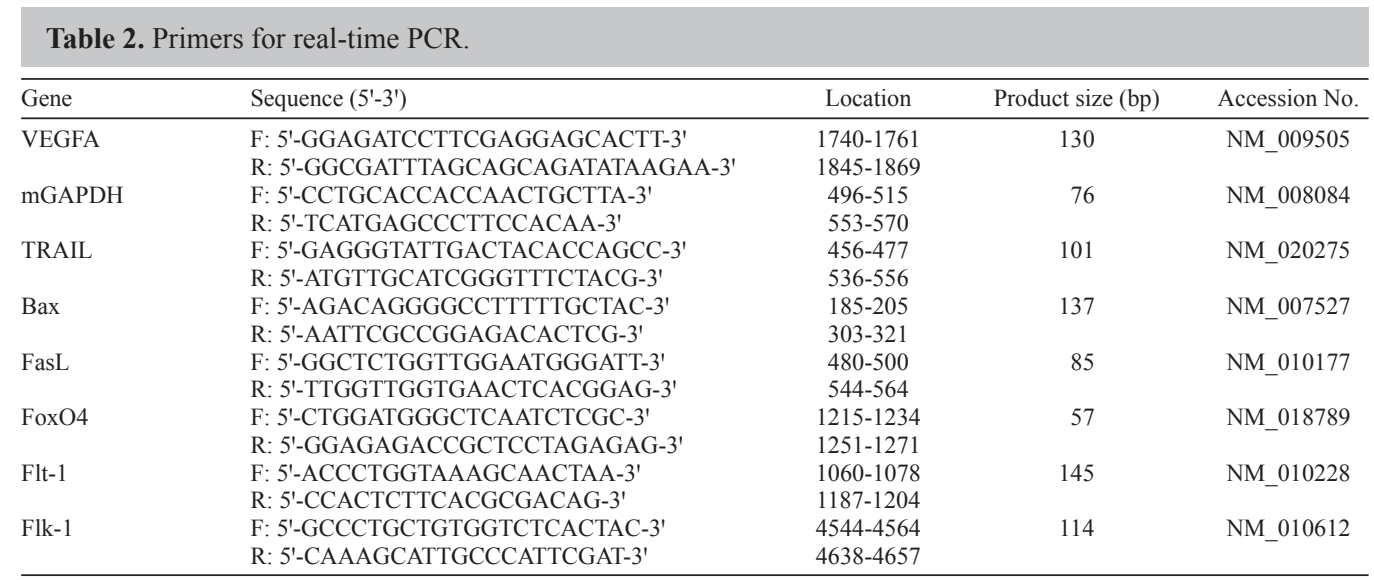

All primers were used at annealing temperature of $60^{\circ} \mathrm{C} . \mathrm{F}=$ forward primer; $\mathrm{R}=$ reverse primer.

\section{ELISA}

The MGC culture supernatants at $48 \mathrm{~h}$ transient transfection were centrifuged for 5 min to remove cells and cell debris. Secreted VEGFA in culture medium was assessed by Solomon Biotechnology Co., China, according to the company procedure.

\section{Western blotting}

Granulosa cells were washed twice with PBS and lysed in $10 \mathrm{mM}$ Tris-HCl, $\mathrm{pH}$ 7.4, $10 \mathrm{mM} \mathrm{NaCl}, 0.5 \% \mathrm{NP}-40,3 \mathrm{mM} \mathrm{MgCl}, 5 \mathrm{mM}$ DTT, and $1 \mathrm{mM}$ PMSF for $1 \mathrm{~h}$ on ice. Nuclei and precipitates were removed by centrifuging the lysates at $14,000 \mathrm{~g}$ for $10 \mathrm{~min}$ at $4^{\circ} \mathrm{C}$. Supernatant protein concentrations were measured using the Bio-Rad protein assay with bovine serum albumin in lysis buffer as the standard and stored at $-80^{\circ} \mathrm{C}$ until analysis of VEGFA expression. Lysate protein, $20 \mu \mathrm{g}$ per lane, equally used for each blot, was separated by SDSPAGE and transferred to a nitrocellulose membrane as described elsewhere (Danforth et al., 2003). A primary anti-VEGFA antibody (Abcam, ab51745, $0.1 \mu \mathrm{g} / \mathrm{mL}$ ) and anti- $\alpha$-tubulin antibody at 1:2000 dilution (Sigma-Aldrich) were used in the Western blotting. After washing three times with TBST (10 min each wash), the membranes were incubated with secondary antibody linked to horseradish peroxidase (HPR) at 1:2000 dilution. HPR activity was detected using Western blot chemiluminescence detection reagents (Promega), according to manufacturer instructions and subsequently exposed to X-ray film and scanned using the Visioneer Paperport software (Visioneer, Inc., Pleasanton, CA, USA). The quantification of VEGFA bands $(27 \mathrm{kDa})$ relative to $\alpha$-tubulin levels was done by densitometry analysis with the Image J software (NIH, Bethesda, MD, USA). 


\section{Construction of lentivirus vector for the delivery of shRNA}

First, the PcmvIE-GFP-SV40 poly-shRNA-U6 cassette was amplified by PCR from VEGF1487 vector (the one that worked efficiently) using the following primers: anti-VEGF-F: 5'-GCTGACTTCTGCAGTCCTGTCGGGTTTC-3', anti-VEGF-R: 5'-AGTACACTCGAGC CCTATCTCGGTCTATT-3'. Primers have incorporated Pst I and XhoI restriction sites (underlined), respectively. PCRs were performed in accordance with standard procedures with a thermostable PrimeSTARTM HS DNA polymerase (TaKaRa) under the following conditions: an initial denaturation at $95^{\circ} \mathrm{C}$ for $4 \mathrm{~min}$, followed by 35 cycles of $98^{\circ} \mathrm{C}$ for $10 \mathrm{~s}, 60^{\circ} \mathrm{C}$ for $15 \mathrm{~s}$, and $72^{\circ} \mathrm{C}$ for $2 \mathrm{~min} 30 \mathrm{~s}$, and a final extension at $72^{\circ} \mathrm{C}$ for 10 min. The PCR products $(2371 \mathrm{bp})$ were separated by $1.0 \%$ agarose gel electrophoresis and stained with ethidium bromide. The band of the expected size was excised and the fragment was recovered with the Gel Extraction Mini kit (Watson Biotechnologies, Inc.). The resulting PCR fragment was digested with Pst I and XhoI enzymes and subcloned into a lentivector backbone. The lentivector backbone was released by Pst $\mathrm{I} / X h o \mathrm{I}$ from pLenti-U6-shRNA (vector was kept in our Laboratory). The final construct was termed pL-VEGF1487.

\section{Production of lentivirus in 293FT cells}

Lentiviruses were generated by transfecting 293FT cells with $9 \mu \mathrm{g}$ ViraPower Packaging mix (Invitrogen) along with $4 \mu \mathrm{g}$ pLenti-U6-shVEGF1487 plasmid. The transfection mix was replaced with fresh culture medium (without antibiotics) at 6-12 $\mathrm{h}$ post-transfection. Supernatant containing infectious lentiviruses was harvested $72 \mathrm{~h}$ post-transfection, centrifuged $\left(3000 \mathrm{rpm} / \mathrm{min}, 15 \mathrm{~min}\right.$, and $\left.4^{\circ} \mathrm{C}\right)$ and filtered through a $0.45-\mu \mathrm{m}$ filter (Millipore). Lentivirus aliquots were prepared and stored at $-80^{\circ} \mathrm{C}$ until use in transductions.

\section{Transduction of MGC with lentivirus vector}

MGC were seeded on 6-well plates at $1 \times 10^{5}$ cells/well and grown overnight prior to transduction. Cells were transduced with $10 \mu \mathrm{g}$ per well virus-containing supernatant from the above procedure. Controls include transduction of MGC with plasmid lacking siRNA (pLeGFP) and transduction with transducing reagent alone (Mock). At $24 \mathrm{~h}$ post-transduction, the medium was changed and replaced with medium without antibiotics. At $48 \mathrm{~h}$, cells were removed using the Trizol ${ }^{\circledR}$ reagent, and RNA was converted to cDNA, and then subjected to qRT-PCR analysis or lysed for Western blotting as described above.

\section{Statistical analysis}

Statistical analyses were performed using the SPSS 13.0 software (SPSS Inc., Chicago, IL, USA). The Student $t$-test was used to test the significance of difference in gene expression level between groups of data. Results are reported as means $\pm \mathrm{SD}$. The level of significance to determine whether treatments were significantly different from controls was $\mathrm{P}<0.05$. 


\section{RESULTS}

\section{Construction of shRNA expression vector against VEGF}

It has been reported that vector-based siRNA can successfully knockdown specific gene expression in mammalian cells, including granulosa cells (Zhang et al., 2003; Kosaka et al., 2007). In this study, we constructed four shRNA expressing plasmids targeting the mouse VEGFA gene (VEGF1051, VEGF1359, VEGF1462, and VEGF1487) with the highest score and an shNC (Table 1) as negative control. All siRNAs constructed formed secondary structures in their antisense strands when checked (Figure 1).

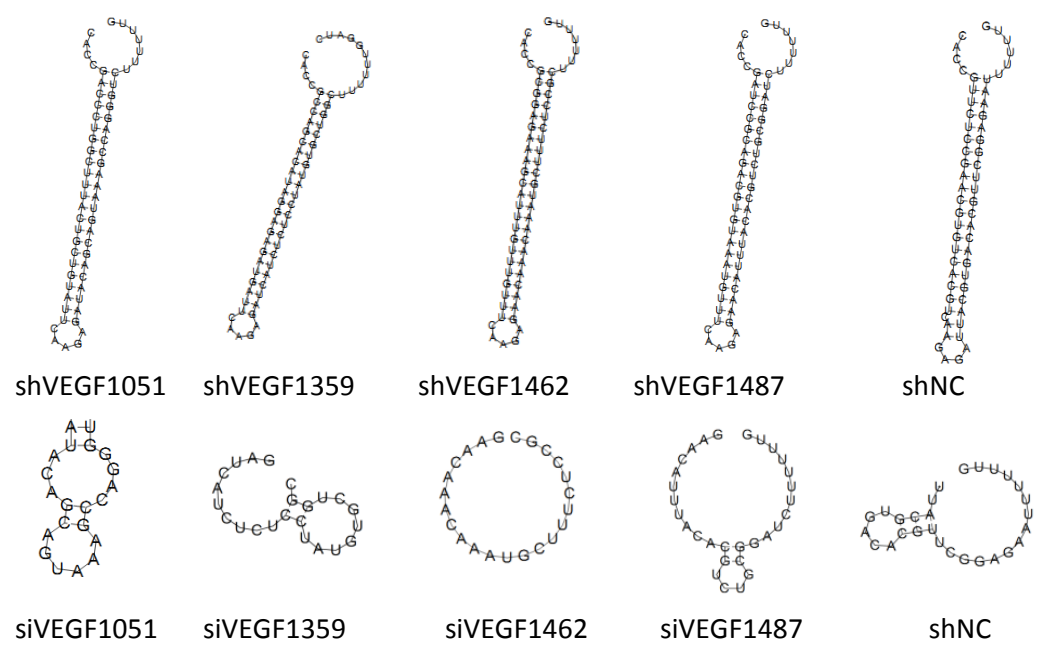

Figure 1. Secondary structures of antisense strands of anti-VEGF siRNAs. shVEGF and siVEGF = short hairpin and short interfering vascular endothelial growth factor, respectively; $\mathrm{shNC}=$ the negative control.

\section{Inhibition of VEGF expression by shRNA plasmid constructs and lentivirus}

The four expression plasmids and shNC were transiently transfected into MGC (Figure 2A) to examine their efficacy in silencing VEGFA expression and cells transfected with transfecting reagent alone (Mock) (Figure 2A). Total RNA was isolated $48 \mathrm{~h}$ post-transfection and converted to cDNA, and the mRNA levels for the different transfected cells were measured using qRT-PCR against the housekeeping gene GAPDH mRNA. All four shRNA plasmids inhibited the expression of the VEGFA gene significantly $(19,46,29$, and $24 \%$, respectively) as compared to shNC and Mock, which showed similar levels of VEGFA expression (Figure 2B). This experiment was repeated several times, and shVEGF1487 was found to be the most stable in its knockdown efficacy; hence, it was subcloned into a lentiviral backbone (Figure $3 \mathrm{~A}$ and $\mathrm{B}$ ) for the production of lentivirus (pL-VEGF1487) and a further 48-h transduction of MGC (Figure 4A), RNA extraction and cDNA production. Using the qRT-PCR procedure described above with Mock cells and pL-eGFP as controls, pL-VEGF1487 also showed a significant knockdown of 52\% in MGC (Figure 4B). A significant proportion of VEGF mRNA may have been from untransfected cells because of the transient transfection procedure. 

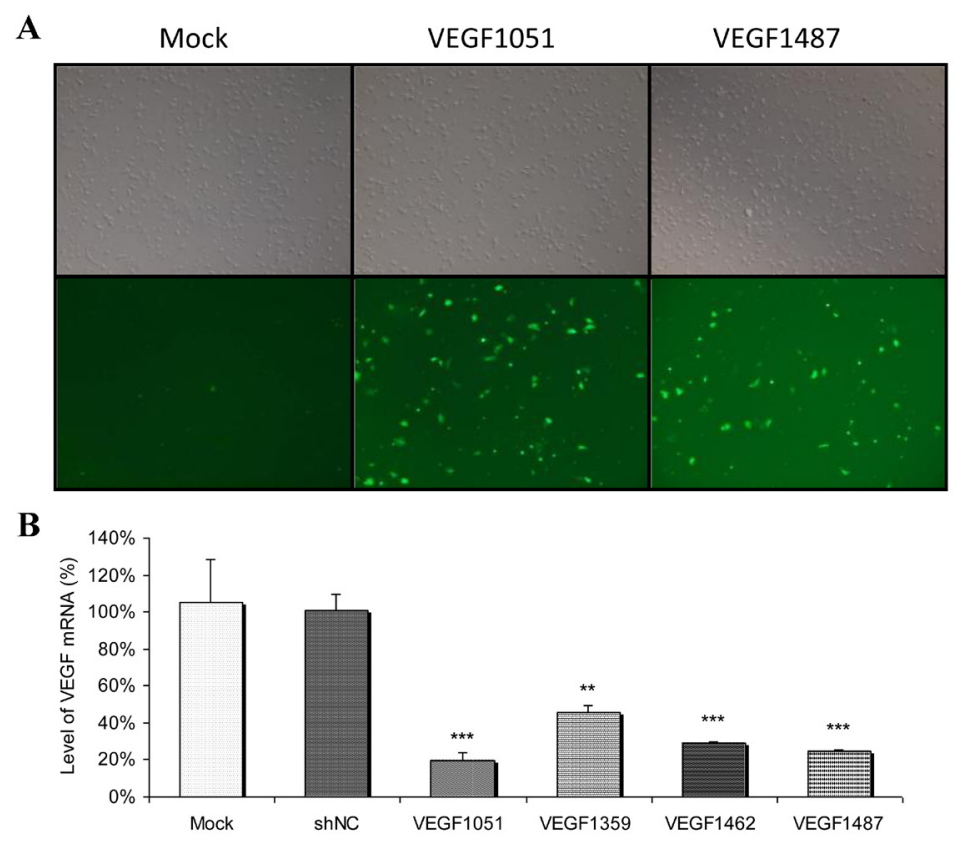

Figure 2. Inhibition of vascular endothelial growth factor (VEGF) expression in mouse granulosa cells (MGC) by shRNA expression vectors. A. Normal resolution and GFP resolution of MGC at 100X magnification of cells transfected for $48 \mathrm{~h}$ on a 6-well plate with $4 \mu \mathrm{g}$ per well transfecting reagent alone (Mock), and two VEGF (other two VEGF were not shown), GFP-positive cells in dark green. B. Analysis of VEGF mRNA levels: shNC, 4 VEGF shRNAs and transfecting reagent alone (Mock) were co-transduced into MGC, and $48 \mathrm{~h}$ after transfection, RNA was extracted and synthesized to cDNA. qRT-PCR analysis was performed to analyze the level of VEGF mRNA. Results are reported as means \pm SEM and are standardized as a percentage of control. ${ }^{* *} \mathrm{P}<0.01$ vs negative control ( $\mathrm{shNC}$ ) and $* * * \mathrm{P}<0.001$ vs negative control (shNC).

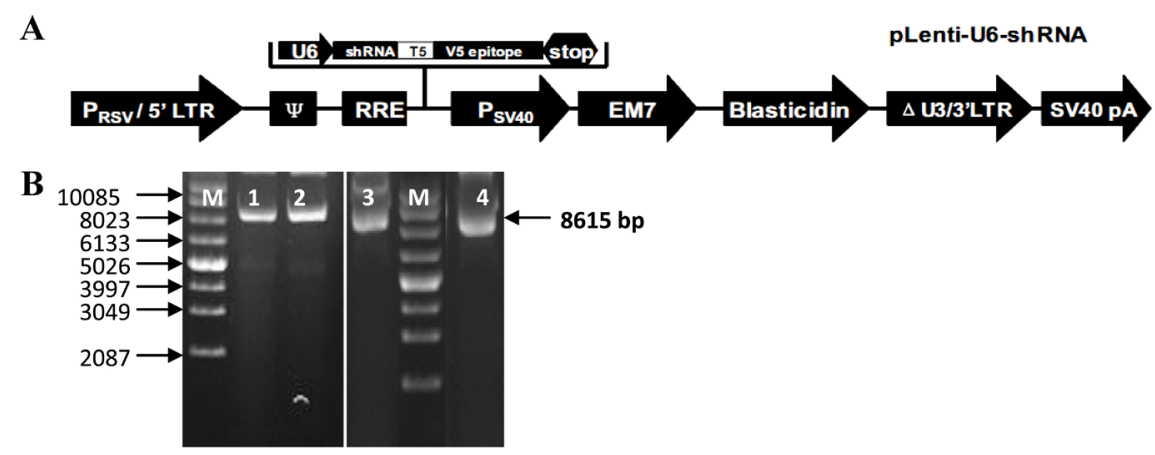

Figure 3. Structure of the pLenti-U6-shVEGF1487 lentiviral transfer vector. A. The pLenti-U6-shVEGF1487 vector expresses the short hairpin form of VEGF siRNA under the control of human U6 promoter and blasticidin resistance gene under the control of SV40 promoter. $\mathrm{P}_{\mathrm{RSV}}=$ RSV enhancer promoter; 5'-LTR = HIV-1 5'-long terminal repeat; $\Psi=$ HIV-1 packaging signal; RRE = HIV-1 Rev response element; PSV40 = SV40 early promoter and origin; EM7 = EM7 promoter; Blasticidin = blasticidin resistance gene; $\Delta \mathrm{U} 3 / 3$ '-LTR $=$ HIV-1 self-inactivating 3 '-LTR; SV40 pA = SV40 polyadenylation signal. B. Agarose gel electrophoresis $(0.8 \%)$ of plasmid pLenti-U6-shRNA. Lane $M=$ molecular marker; lane 1=pLV-VEGF1051; lane 2=pLV-VEGF1462; lane 3 = pLV-VEGF1359; lane 4=pLV-VEGF1487. 
A

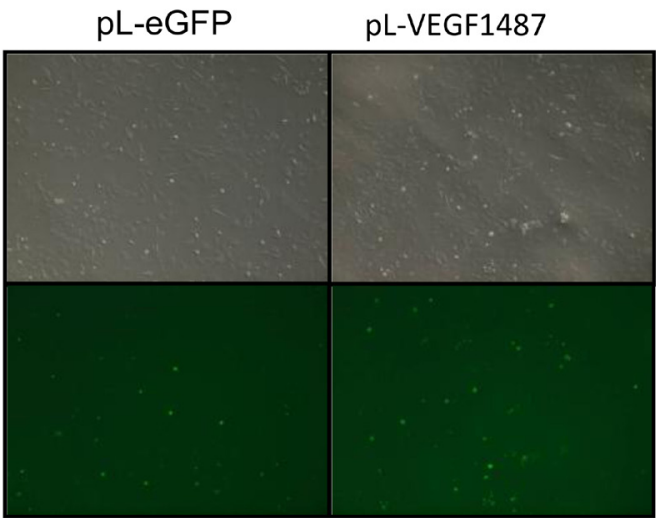

B

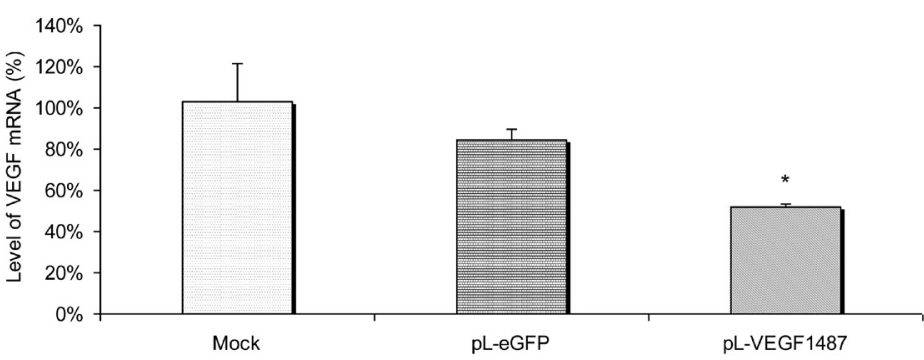

Figure 4. Effective inhibition of vascular endothelial growth factor (VEGF) expression in mouse granulosa cells (MGC) by using lentivectors for shRNA delivery. A. Normal resolution and GFP resolution of MGC at 100X magnification of cells transduced for $48 \mathrm{~h}$ on a 6 -well plate with $10 \mu \mathrm{L}$ of virus containing supernatant per well transduction reagent alone (Mock) and pL-eGFP as controls and pL-VEGF1487, GFP-positive cells in dark green. B. Analysis of VEGF mRNA levels. pL-eGFP and pL-VEGF1487 shRNAs and transduction reagent alone (Mock) were co-transduced into MGC, and $48 \mathrm{~h}$ after transduction, RNA was extracted to produce cDNA. qRT-PCR analysis was performed to analyze the level of VEGF mRNA. Results are reported as means \pm SEM and are standardized as a percentage of control. *P $<0.05 v s$ negative control ( $\mathrm{pL}-\mathrm{eGFP})$.

\section{Effect of shRNA plasmid constructs on VEGF receptor gene expression}

VEGF receptor genes (Flt-1 and Flk-1/KDR) have also been shown to be expressed in granulosa cells (Einspanier et al., 2002; Greenaway et al., 2004). Hence, we investigated the effects of the shRNAs on both Flt-1 and Flk-1 mRNA levels, against housekeeping GAPDH mRNA, and found that there was significant knockdown of all shRNA constructs compared to the shNC (Figure 5A and B). shVEGF1359 had a knockdown efficacy of 3\%, while the other three shVEGF showed a knockdown below $0 \%$ in MGC for both receptor genes.

\section{Effect of shRNA plasmid constructs on VEGF apoptosis gene expression}

The effects of VEGFA expression on MGC apoptosis were also determined by investigating the mRNA level of five VEGFA apoptosis genes (Bax, FasL, TRAIL, and FoxO4) against the housekeeping gene GAPDH mRNA. It was found that the expression of these genes was also significantly knocked down compared to the shNC for most of the shRNA constructs except for FoxO4, which showed no significant inhibitory effect in shVEGF1359 (111\%) (Figure 6). 

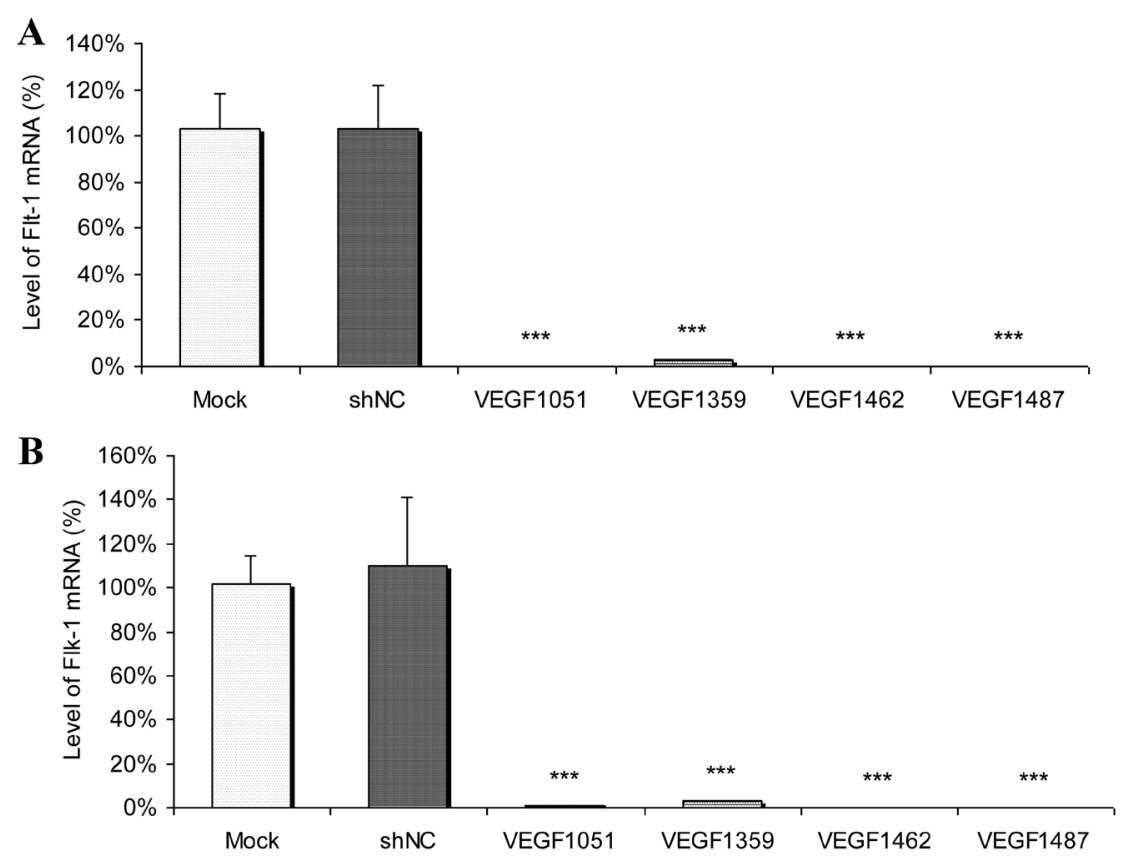

Figure 5. Inhibition of vascular endothelial growth factor (VEGF) receptor gene expression in mouse granulosa cells (MGC) by shRNA expression vectors. A. Analysis of the Flt-1 mRNA levels. B. Analysis of the Flk-1 mRNA levels. shNC, 4 VEGF shRNAs and transfecting reagent alone (Mock) were transfected into MGC on a 6-well plate at $4 \mu \mathrm{g}$ per well, and $48 \mathrm{~h}$ after transfection, RNA was extracted to produce cDNA. qRT-PCR analysis was performed to analyze the level of Flt- 1 and Flk-1 mRNA. Results are reported as means \pm SEM and are standardized as a percentage of control. $* * * \mathrm{P}<0.001$ vs negative control (shNC).

\section{Effect of shRNA plasmid constructs and lentivirus on VEGF protein levels}

Similar to the above experimental protocol, MGC was transfected with the shRNA expression plasmids and the conditioned media was used to perform ELISA (Solomon Biotechnology, Co.). This was to quantify the amount of VEGF protein present in the conditioned media for MGC transfected with the four shRNAs constructs, shNC and Mock cells. There was significant knockdown efficacy in the level of VEGFA protein concentration in the culture media for the four shVEGF compared to the controls (Figure 7A). These results were in agreement with those analyzed by qRT-PCR experiments for mRNA levels of VEGFA, VEGF receptor genes and apoptosis genes.

VEGFA protein levels were measured by Western blotting in lysates collected from MGC transfected for $48 \mathrm{~h}$ with the four shRNA constructs, shNC and Mock cells, as well as lysates from MGC transduced for $48 \mathrm{~h}$ with lentiviral vector pL-VEGF1487 and Mock cells used as control. All wells were loaded with an equal amount of protein. Densitometric analysis of VEGFA expression level was performed by calculating the band intensity of the purified VEGFA protein for experimental groups versus $\alpha$-tubulin. Protein concentration was found to be higher only in shVEGF1359 versus shNC but 17\% lower versus Mock, which suggests 
that there may be some levels of protein in the supernatant before the cells were lysed. All other shVEGFs [VEGF1051 (43\%), VEGF1462 (59\%), and VEGF1487 (64\%)], as well as pL-VEGF1487 with 86\%, expressed lower levels of protein as compared to shNC and Mock (Figure 7B and C) with similar trends as in the qRT-PCR results. These data indicated that VEGF expression can be strongly inhibited at both the mRNA and protein levels by vectorbased RNAi in agreement with Zhang et al. (2008).
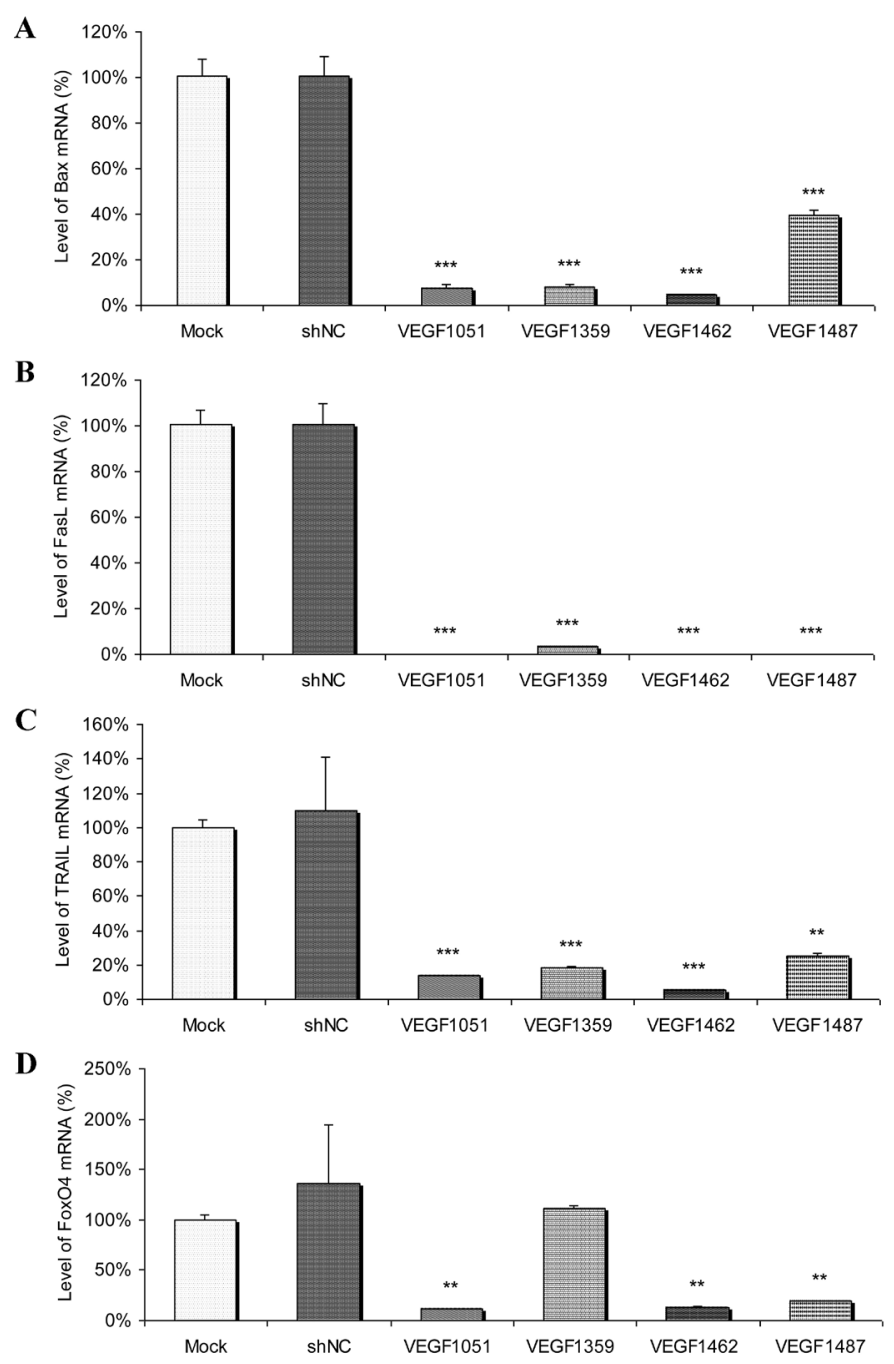

Figure 6. Inhibition of vascular endothelial growth factor (VEGF) apoptosis gene expression in mouse granulosa cells (MGC) by shRNA expression vectors. A. Analysis of the Bax mRNA levels. B. Analysis of the FasL mRNA levels. C. Analysis of the TRAIL mRNA levels. D. Analysis of the FoxO4 mRNA levels. shNC, 4 VEGF shRNAs and transfecting reagent alone (Mock) were transfected into MGC on a 6-well plate at $4 \mu \mathrm{g}$ per well, and $48 \mathrm{~h}$ after transfection, RNA was extracted to produce cDNA. qRT-PCR analysis was performed to analyze the level of Bax, FasL, TRAIL, and FoxO4 mRNA. Results are reported as means \pm SEM and are standardized as a percentage of control. $* * \mathrm{P}<0.01$ vs negative control $(\mathrm{shNC})$ and $* * * \mathrm{P}<0.001$ vs negative control (shNC). 


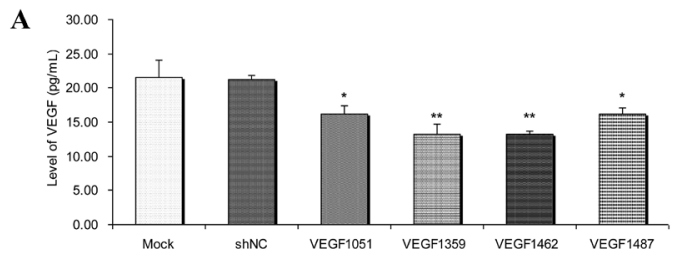

B

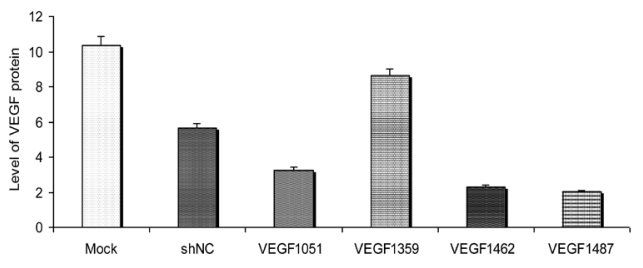

VEGF

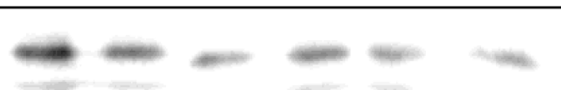

$\alpha$-tubulin

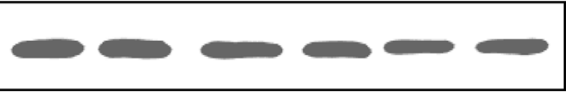

C

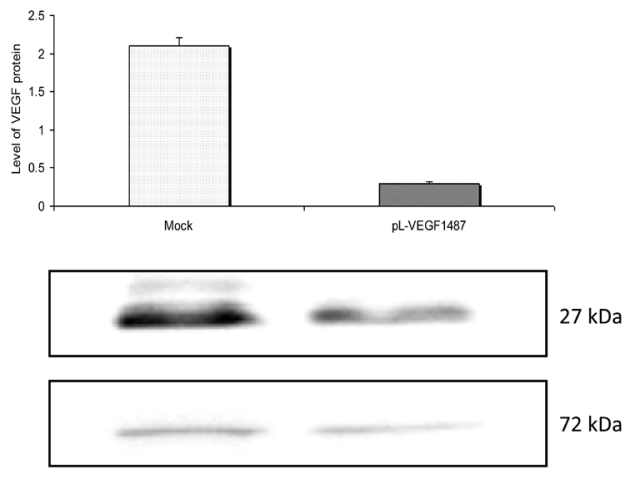

Figure 7. Protein expression level. A. shNC, 4 VEGF shRNAs and transfecting reagent alone (Mock) at $4 \mu \mathrm{g}$ per well on a 6-well plate were transfected into mouse granulosa cells (MGC), and $48 \mathrm{~h}$ after transfection, protein concentration in the MGC culture media was analyzed by ELIZA. Results are reported as means \pm SEM and are standardized as a percentage of control. $* \mathrm{P}<0.05$ vs negative control (shNC) and $* * \mathrm{P}<0.01$ vs negative control (shNC). B. Western blot analysis of MGC transfected for $48 \mathrm{~h}$ with $4 \mu \mathrm{g}$ per well on a 6 -well plate; shVEGF interfering vector, shNC and transfecting reagent alone (Mock) as control. C. Western blot analysis of MGC transduced for $48 \mathrm{~h}$ with $10 \mu \mathrm{g}$ per well on a 6-well plate, lentiviral vector and transfecting reagent alone (Mock) as control.

\section{DISCUSSION}

In this research, we present a strategy to knockdown the VEGFA gene in MGC using shRNA expression plasmids and lentiviral vector RNAi. This method provides a novel tool to study the function of VEGFA and its receptors and apoptosis genes of MGC in vitro. Previous reports have indicated that VEGF and its receptors, VEGFR-1 and VEGFR-2, are expressed in pig follicle cells (Barboni et al., 2000), bovine granulosa cells (Einspanier et al., 2002; Greenaway et al., 2004) and MGC (Wulff et al., 2002) in a coordinated fashion in which they protect these cells from apoptosis, which demonstrates that VEGF functions as a survival factor for ovarian follicle and granulosa cells (Kosaka et al., 2007). Therefore, the inhibition of the VEGF gene by RNAi strategy to study its function in MGC requires an understanding of the related effects of this strategy downstream of the VEGF-related genes.

Our study showed that RNAi using a plasmid-based and lentivector strategy can stably silence VEGFA in MGC. We first constructed four plasmid-based expression systems, targeting the VEGFA gene and transfected MGC to test the efficacy of the vector by qRT-PCR measurement of mRNA levels of VEGF and its receptor genes and apoptosis genes, and found that all constructs demonstrated successful knockdown of the genes. Hence, we further checked the effects on the protein level of the transfected culture medium, by ELISA, and on protein level in MGC by Western blotting and found that both results were in agreement, except only for 
VEGF1359, which showed higher levels of protein that might have been in the supernatant before cells were lysed. Repeated experiments indicated that shVEGF1487 was the most stable in its knockdown efficiency and was thus chosen for further lentiviral production. This was done by transcribing the sense and antisense strands of anti-VEGF siRNA into hairpin structures under the control of the U6 polymerase III promoter (Zhang et al., 2008) and then processed into a lentiviral backbone. This lentiviral vector was transduced in MGC, and VEGF mRNA levels and protein level results were also in agreement with results of transduction of MGC by expression plasmid compared to the control group (Figure 4B). These results demonstrated that the current VEGF siRNA sequence can completely inhibit VEGFA mRNA expression in MGC.

Unlike VEGF, which is produced by most cell types, research has previously demonstrated that the VEGF receptors Flt-1 and Flk-1 are coexpressed in vascular cells (Ferrara, 2002) including granulosa cells in which they interact to protect these cells against apoptotic cell death, and inhibition of VEGF causes a decrease in Flt and Flk expression (Greenaway et al., 2004; Kosaka et al., 2007). Flt-1 is believed to be expressed in ovarian follicles that consist of oocyte, granulosa cells and the cells of the internal and external theca layer (Okamura et al. 2001). Therefore, in this research after successful knockdown of the VEGFA gene by shRNA expression plasmids, we checked the mRNA level of Flt-1 and Flk-1 and found similar inhibition of over $97 \%$ knockdown efficiency. This further supports the hypothesis that siRNAs with specific sequences can be an effective tool to inhibit related genes downstream of a target gene.

VEGF is also known to be involved in the regulation of granulosa cell apoptosis when its treatment was found to reduce the incidence of apoptosis and activate caspase- 3 expression in granulosa cells but not fibroblast cells, because of the absence of VEGF receptors (Greenaway et al., 2004). Apoptosis is known to be regulated by two ways so far, by targeting the functions of the mitochondrion or using adapter proteins to transduce signals to the apoptotic mechanism. Bax is said to be responsible for the formation of pores on the mitochondrion by regulating the voltage-dependent anion channel. Our result showing significant inhibition of Bax by VEGFA knockdown was contrary to findings of Kosaka et al. (2007), who showed no significant difference. The tumor suppressor protein $\mathrm{p} 53$, which has a transcription-independent role in apoptosis, also interacts with Bax to promote its activation as well as insertion into the mitochondrial membrane. Trimeric receptor with bound specific ligands such as FasL and TRAIL is an adapter protein that leads to the formation of the death-inducing signaling complex, which contains the FADD, caspase-8 and caspase-10 (Chang et al. 1998). Therefore, inhibition of the binding of these ligands, as our result indicates, would also inhibit caspase expression downstream, which suggests that VEGFA is also associated with the mitochondrial pathway. Forkhead transcription factors (FoxO) are a family of proteins found in the cytoplasm and nucleus of cells, which function as sensors of signaling pathways and play important roles in cell cycle arrest, apoptosis, angiogenesis, stress resistance, and energy metabolism, through the regulation of gene expression (Accili and Arden, 2004). Previous research has indicated that nuclear expression of FoxO4 suppresses VEGF in the P13K/AKT pathway (Tang and Lasky, 2003). Our results indicated that the inhibition of VEGF expression also reduces FoxO4 gene expression, which demonstrates that FoxO4 plays an important role in signal regulation of VEGF through the P13K/AKT pathway.

In conclusion, these findings suggest that RNAi mediated by shVEGF expression plasmids and lentivector is a promising tool for inhibiting the gene expression of VEGFA of the VEGF family, its related receptors and apoptosis components in MGC in vitro. Cell trans- 
fection and transduction by expression plasmids and lentiviral vectors, respectively, resulted in selective inhibition of VEGFA gene expression, leading to knockdown of VEGF receptor genes and apoptosis genes and reduction in protein levels when checked both in the culture medium and MGC. This research indicates that RNAi with lentiviral vector can be used to study and understand the relationship of different VEGF family members or specific isoforms within the VEGF gene and genes of their related receptors or apoptosis genes in MGC in vitro. Presently, research is in progress to further study follicle development in vivo with the shRNA packaged in a tissue-specific lentiviral vector.

\section{ACKNOWLEDGMENTS}

Research supported by the National Special Foundation for Transgenic Species of China (Grant \#2008ZX08006-003). The authors thank all their classmates and teachers for their helpful discussions and technical assistance.

\section{REFERENCES}

Abramovich D, Irusta G, Parborell F and Tesone M (2010). Intrabursal injection of vascular endothelial growth factor trap in eCG-treated prepubertal rats inhibits proliferation and increases apoptosis of follicular cells involving the PI3K/ AKT signaling pathway. Fertil. Steril. 93: 1369-1377.

Accili D and Arden KC (2004). FoxOs at the crossroads of cellular metabolism, differentiation, and transformation. Cell 117: 421-426.

Barboni B, Turriani M, Galeati G, Spinaci M, et al. (2000). Vascular endothelial growth factor production in growing pig antral follicles. Biol. Reprod. 63: 858-864.

Brummelkamp TR, Bernards R and Agami R (2002). A system for stable expression of short interfering RNAs in mammalian cells. Science 296: 550-553.

Bruno JB, Celestino JJ, Lima-Verde IB, Lima LF, et al. (2009). Expression of vascular endothelial growth factor (VEGF) receptor in goat ovaries and improvement of in vitro caprine preantral follicle survival and growth with VEGF. Reprod. Fertil. Dev. 21: 679-687.

Celik-Ozenci C, Akkoyunlu G, Kayisli UA, Arici A, et al. (2003). Localization of vascular endothelial growth factor in the zona pellucida of developing ovarian follicles in the rat: a possible role in destiny of follicles. Histochem. Cell Biol. 120: 383-390.

Chang HY, Nishitoh H, Yang X, Ichijo H, et al. (1998). Activation of apoptosis signal-regulating kinase 1 (ASK1) by the adapter protein Daxx. Science 281: 1860-1863.

Danforth DR, Arbogast LK, Ghosh S, Dickerman A, et al. (2003). Vascular endothelial growth factor stimulates preantral follicle growth in the rat ovary. Biol. Reprod. 68: 1736-1741.

Doyle LK, Walker CA and Donadeu FX (2010). VEGF modulates the effects of gonadotropins in granulosa cells. Domest. Anim. Endocrinol. 38: 127-137.

Einspanier R, Schonfelder M, Muller K, Stojkovic M, et al. (2002). Expression of the vascular endothelial growth factor and its receptors and effects of VEGF during in vitro maturation of bovine cumulus-oocyte complexes (COC). Mol. Reprod. Dev. 62: 29-36.

Elbashir SM, Harborth J, Lendeckel W, Yalcin A, et al. (2001). Duplexes of 21-nucleotide RNAs mediate RNA interference in cultured mammalian cells. Nature 411: 494-498.

Elbashir SM, Harborth J, Weber K and Tuschl T (2002). Analysis of gene function in somatic mammalian cells using small interfering RNAs. Methods 26: 199-213.

Ferrara N (2002). VEGF and the quest for tumour angiogenesis factors. Nat. Rev. Cancer 2: 795-803.

Giering JC, Grimm D, Storm TA and Kay MA (2008). Expression of shRNA from a tissue-specific pol II promoter is an effective and safe RNAi therapeutic. Mol. Ther. 16: 1630-1636.

Greenaway J, Connor K, Pedersen HG, Coomber BL, et al. (2004). Vascular endothelial growth factor and its receptor, Flk1/KDR, are cytoprotective in the extravascular compartment of the ovarian follicle. Endocrinology 145: 2896-2905.

Hannon GJ (2002). RNA interference. Nature 418: 244-251. 
Hicklin DJ and Ellis LM (2005). Role of the vascular endothelial growth factor pathway in tumor growth and angiogenesis. J. Clin. Oncol. 23: 1011-1027.

Irusta G, Abramovich D, Parborell F and Tesone M (2010). Direct survival role of vascular endothelial growth factor (VEGF) on rat ovarian follicular cells. Mol. Cell Endocrinol. 325: 93-100.

Klagsbrun M and D'Amore PA (1996). Vascular endothelial growth factor and its receptors. Cytokine Growth Factor Rev. 7: 259-270.

Kosaka N, Sudo N, Miyamoto A and Shimizu T (2007). Vascular endothelial growth factor (VEGF) suppresses ovarian granulosa cell apoptosis in vitro. Biochem. Biophys. Res. Commun. 363: 733-737.

Lee NS, Dohjima T, Bauer G, Li H, et al. (2002). Expression of small interfering RNAs targeted against HIV-1 rev transcripts in human cells. Nat. Biotechnol. 20: 500-505.

McManus MT and Sharp PA (2002). Gene silencing in mammals by small interfering RNAs. Nat. Rev. Genet. 3: 737-747.

Okamura Y, Myoumoto A, Manabe N, Tanaka N, et al. (2001). Protein tyrosine kinase expression in the porcine ovary. Mol. Hum. Reprod. 7: 723-729.

Paul CP, Good PD, Winer I and Engelke DR (2002). Effective expression of small interfering RNA in human cells. Nat. Biotechnol. 20: 505-508.

Petersen CP, Bordeleau ME, Pelletier J and Sharp PA (2006). Short RNAs repress translation after initiation in mammalian cells. Mol. Cell 21: 533-542.

Reynolds A, Leake D, Boese Q, Scaringe S, et al. (2004). Rational siRNA design for RNA interference. Nat. Biotechnol. 22: 326-330.

Shen HL, Xu W, Wu ZY, Zhou LL, et al. (2007). Vector-based RNAi approach to isoform-specific downregulation of vascular endothelial growth factor (VEGF)165 expression in human leukemia cells. Leuk. Res. 31: 515-521.

Shi Y (2003). Mammalian RNAi for the masses. Trends Genet. 19: 9-12.

Tamanini C and De Ambrogi M (2004). Angiogenesis in developing follicle and corpus luteum. Reprod. Domest. Anim. 39: 206-216.

Tang TT and Lasky LA (2003). The forkhead transcription factor FOXO4 induces the down-regulation of hypoxiainducible factor 1 alpha by a von Hippel-Lindau protein-independent mechanism. J. Biol. Chem. 278: 30125-30135.

Terman BI and Dougher-Vermazen M (1996). Biological properties of VEGF/VPF receptors. Cancer Metastasis Rev. 15: 159-163.

Wolters NM and Mackeigan JP (2008). From sequence to function: using RNAi to elucidate mechanisms of human disease. Cell Death Differ. 15: 809-819.

Wulff C, Wilson H, Wiegand SJ, Rudge JS, et al. (2002). Prevention of thecal angiogenesis, antral follicular growth, and ovulation in the primate by treatment with vascular endothelial growth factor Trap R1R2. Endocrinology 143: 2797-2807.

Yoo JY, Kim JH, Kwon YG, Kim EC, et al. (2007). VEGF-specific short hairpin RNA-expressing oncolytic adenovirus elicits potent inhibition of angiogenesis and tumor growth. Mol. Ther. 15: 295-302.

Zhang GY, Yi CG, Li X, Zheng Y, et al. (2008). Inhibition of vascular endothelial growth factor expression in keloid fibroblasts by vector-mediated vascular endothelial growth factor shRNA: a therapeutic potential strategy for keloid. Arch. Dermatol. Res. 300: 177-184.

Zhang L, Yang N, Mohamed-Hadley A, Rubin SC, et al. (2003). Vector-based RNAi, a novel tool for isoform-specific knockdown of VEGF and anti-angiogenesis gene therapy of cancer. Biochem. Biophys. Res. Commun. 303: 1169-1178. 